\title{
Linear Response Calculations of Lattice Dynamics in Strongly Correlated Systems
}

\author{
S. Y. Savrasov \\ Department of Physics, New Jersey Institute of Technlogy, Newark, NJ 07102 \\ G. Kotliar \\ Department of Physics and Astronomy, Rutgers University, Piscataway, NJ 08854
}

(April 2002)

\begin{abstract}
We introduce a new linear response method to study the lattice dynamics of materials with strong correlations. It is based on a combination of dynamical mean field theory of strongly correlated electrons and the local density functional theory of electronic structure of solids. We apply the method to study the phonon dispersions of a prototype Mott insulator NiO. Our results show overall much better agreement with experiment than the corresponding local density predictions.
\end{abstract}

71.27.+a, 63.10.+a, 71.15.-m

Computational studies of lattice dynamics and structural stability in strongly correlated electronic systems is a challenging theoretical problem. In the past density functional theory (DFT) in its local density or generalized gradient approximations (LDA or GGA) [1] has delivered the full lattice dynamical information and electron-phonon related properties of a variety of simple metals, transition metals, as well as semiconductors with exceptional accuracy [2]. This is mainly due to the introduction of a linear response approach [3, 4 . This method overcame the problems of traditional techniques based on static susceptibility calculations which generally fail to reproduce lattice dynamical properties of real materials due to difficulties connected with the summations in high-energy states and the inversion of very large dielectric matrix [5].

Despite these impressive successes, there is by now clear evidence that the present methodology fails when applied to strongly correlated materials. For example, the local density predictions for such properties as bulk modulus and elastic constants in metallic Plutonium are approximately one order of magnitude off from experiment [6]; the phonon spectrum of Mott Insulators such as $\mathrm{MnO}$ is not predicted correctly by LDA [7].

In this work we describe a new linear response method to study the lattice dynamics of correlated materials. It is based on the dynamical mean field theory (DMFT) [8], a many-body technique developed to study systems with strong on-site Coulomb repulsion. Recent progress in merging [9] this many-body description with the realistic LDA based electronic structure calculations has already led to new insights in long standing problems, such as, the temperature dependence of the magnetic properties of $\mathrm{Fe}$ and $\mathrm{Ni} 10$, the volume collapse transition in $\mathrm{Ce}$ [11], and $\mathrm{Pu}$ 12,13]. We generalize this LDA+DMFT method to carry out linear response calculations by finding self-consistent changes in both charge densities and local Green functions induced by atomic displacements.

The LDA+DMFT approach computes the total energy, the charge density and the local spectral function of the correlated electrons simultaneously. The latter spectra are known to be quite different for a strongly correlated system such, e.g., as heavy fermion metal, from the Kohn Sham spectra of the local density functional theory due to the appearance of strongly renormalized quasiparticle features at the Fermi level and lower and upper Hubbard features or satellites at higher energies [8]. The LDA+DMFT technique can therefore provide a link between the photoemission spectra and the lattice dynamics of correlated materials, which is an interesting open problem.

As a test we consider the prototype Mott insulator $\mathrm{NiO}$. In this material at low temperatures, the static Hartree-Fock limit of our formulation which is formally equivalent to the $\mathrm{LDA}+\mathrm{U}$ method [14] can be used. Results for the phonon dispersion and the static dielectric properties of this material are found in much better agreement with the experiment than the results of corresponding LDA based calculations. We discuss how correlations affect the calculated dielectric constants and the Born effective charges using the linear-response method.

Our approach considers both the charge density $\rho$ and the local Green function $\hat{G}(\omega)$ as parameters of a spectral density functional [13]. To find its extremum, a set of Dyson equations is solved self-consistently:

$$
\left(-\nabla^{2}+V_{e f f}+\hat{\Sigma}(\omega)-\hat{\Sigma}_{d c}-\epsilon_{\mathbf{k} j \omega}\right) \psi_{\mathbf{k} j \omega}^{r}=0
$$

where $V_{\text {eff }}$ is the effective potential of the local density functional and $\hat{\Sigma}(\omega)$ is the local self-energy operator. Since the local density approximation contains an average correlation energy, a double counting term $\hat{\Sigma}_{d c}$ appears in the equation (1). Both $V_{\text {eff }}$ and $\hat{\Sigma}(\omega)$ are functionals of the density and the local Green function which can be found using the formula:

$$
\hat{G}(\omega)=\sum_{\mathbf{k} j} \frac{\psi_{\mathbf{k} j \omega}^{l} \psi_{\mathbf{k} j \omega}^{r}}{\epsilon_{\mathbf{k} j \omega}-\omega}
$$

Note, that the Greens function is a non-hermitian matrix that is frequency dependent so both the eigenvalues 
$\epsilon$ and right/left eigenvectors $\psi^{r, l}$ are treated formally as frequency dependent quantities: $\epsilon_{\mathbf{k} j \omega}, \psi_{\mathbf{k} j \omega}^{r}, \psi_{\mathbf{k} j \omega}^{l}$. (The latter satisfies the Dyson equation (11) with the wave function placed on the left.) In practice [9, 12], (11) is solved on the Matsubara axis for a finite set of imaginary frequencies $i \omega_{n}$ using some localized orbital representation such, e.g., as linear muffin-tin orbitals (LMTOs) 15 $\chi_{\alpha}^{\mathbf{k}}$ for the eigenvectors $\psi_{\mathbf{k} j \omega}^{r}$

$$
\psi_{\mathbf{k} j \omega}^{r}=\sum_{\alpha} A_{\alpha}^{\mathbf{k} j \omega} \chi_{\alpha}^{\mathbf{k}}
$$

which substitutes the differential equation (11) by a matrix eigenvalue problem.

Once the local Green function is constructed, the new charge density, effective potential and the local selfenergy are computed. The latter is found by solving the Anderson impurity model using a suitable manybody technique. This entire formulation requires the selfconsistent procedure which delivers the total energy of the interacting electronic system.

The dynamical matrix is the second order derivative of the energy. As with the ordinary density functional formulation of the problem [16], we deal with the first order corrections to the charge density, $\delta \rho$, as well as the first order correction to the local Greens function $\delta \hat{G}(\omega)$ which should be considered as two independent variables in the functional of the dynamical matrix. To find the extremum, a set of the linearized Dyson equations has to be solved self-consistently:

$$
\begin{aligned}
\left(-\nabla^{2}+\right. & \left.V_{e f f}+\hat{\Sigma}(\omega)-\hat{\Sigma}_{d c}-\epsilon_{\mathbf{k} j \omega}\right) \delta \psi_{\mathbf{k} j \omega}^{r}+ \\
& +\left(\delta V_{e f f}+\delta \hat{\Sigma}(\omega)-\delta \hat{\Sigma}_{d c}\right) \psi_{\mathbf{k} j \omega}^{r}=0
\end{aligned}
$$

which leads us to consider the first order changes in the effective potential $\delta V_{\text {eff }}$ and in the local self-energy operator $\delta \hat{\Sigma}(\omega)$. Here and in the following we will assume that the phonon wave vector of the perturbation $\mathbf{q}$ is different from zero, and, therefore, the first order changes in the eigenvalues $\delta \epsilon_{\mathbf{k} j \omega}$ drop out. The quantities $\delta V_{\text {eff }}$ and $\delta \hat{\Sigma}(\omega)$ are the functionals of $\delta \rho$ and $\delta \hat{G}(\omega)$ and should be found self-consistently. In particular, the change in the self-energy $\delta \hat{\Sigma}(\omega)$ assumes the development of solving an Anderson impurity model linearized with respect to atomic displacements.

In practice, change in the eigenvector $\delta \psi_{\mathbf{k} j \omega}$ has to be expanded in some basis set. Previous linear response schemes were based on tight-binding methods [17], plane wave pseudopotentials [3, 4, 18, 19], linear augmented plane waves [20], mixed orbitals [21] and linear muffin-tin orbitals [22]. To build an effective computational scheme applicable for systems with localized orbitals we use LMTO representation as the basis. Due to its explicit dependence on the atomic positions both Hellmann-Feynman contributions and incomplete basis set corrections [23] appear in the expression for the dynamical matrix 116]. We expand $\delta \psi_{\mathbf{k} j \omega}$ as follows

$$
\delta \psi_{\mathbf{k} j \omega}=\sum_{\alpha}\left\{\delta A_{\alpha}^{\mathbf{k} j \omega} \chi_{\alpha}^{\mathbf{k}+\mathbf{q}}+A_{\alpha}^{\mathbf{k} j \omega} \delta \chi_{\alpha}^{\mathbf{k}}\right\}
$$

where we introduced both changes in the frequency dependent variational coefficients $\delta A_{\alpha}^{\mathbf{k} j \omega}$ as well as changes in the basis functions $\delta \chi_{\alpha}^{\mathbf{k}}$. The latter helps us to reach convergency in the entire expression (5) with respect to the number of the basis functions $\{\alpha\}$ fast since the contribution with $\delta \chi_{\alpha}^{\mathbf{k}}$ takes into account all rigid movements of the localized orbitals [22].

The first-order changes in the Green function can be found as follows

$$
\delta \hat{G}(\omega)=\sum_{\mathbf{k} j} \frac{\delta \psi_{\mathbf{k} j \omega}^{l} \psi_{\mathbf{k} j \omega}^{r}+\psi_{\mathbf{k} j \omega}^{l} \delta \psi_{\mathbf{k} j \omega}^{r}}{\epsilon_{\mathbf{k} j \omega}-\omega}
$$

which should be used to evaluate the first order change in the charge density and the dynamical matrix itself.

Despite formal complexity of the outlined method we see two significant simplifications which can be implemented in practice. First, using static limit of the dynamical mean field theory leads to a frequency independent approximation for $\hat{\Sigma}$. This recovers the LDA+U method and its linear-response analog for the present problem. Second, it is generally expected that only low frequency behavior of the self-energy will drastically affect the static electronic response, since it modifies the one-electron spectrum near the Fermi level. If the Hubbard subbands are separated by sufficiently large $U$ they are essentially quasi-atomic features and may be though as the states tightly bound and moving rigidly as the atom vibrates. These two simplified calculational schemes may be explored for the lattice dynamics in correlated materials without significant complications.

We now describe our implementation of the method for calculating vibrational spectrum of the prototype Mott insulator NiO. It is well known 24] that the LDA underestimates both the value of the energy gap and the magnetic moment of this material. On the other hand, the use of the LDA+U method fixes both problems 24] when using non-zero Hubbard parameter $U$. The use of the $\mathrm{LDA}+\mathrm{U}$ approximation is equivalent here to a static limit of the full frequency resolved theory outline above: the self-energy $\hat{\Sigma}(\omega)$ becomes an orbital-dependent correction to the Kohn-Sham potential $V_{\text {eff }}$ expressed via the density matrix of the localized electrons, $n_{\alpha \beta}$, and the change in the self-energy, $\delta \hat{\Sigma}$, is expressed via the changes in occupation numbers, $\delta n_{\alpha \beta}$, due to atomic movements. Here we will assume that the on-site Hubbard interaction $U$ is fixed for all the displacements. The use of the static approximation significantly simplifies our implementation: the solution of the impurity model and its change with respect to the displacements collapses and the quantities which have to be found self-consistently are the changes in charge density $\delta \rho$ and in the occupancy matrix $\delta n_{\alpha \beta}$. The latter substitutes finding full change $\delta \hat{G}(\omega)$ in the local Green function of the system. 
We have calculated the phonon dispersions of $\mathrm{NiO}$ within the LDA and the static limit of DMFT. We utilize $2 \kappa$ LMTO basis set for these linear response calculations assuming antiferromagnetic spin alignment at $\mathrm{Ni}$ sites. We use special point technique for integrating over the Bighldurfaycasevr Kołliarhding to a $(6,6,6)$ grid and experimental lattice parameter $a=7.926$ a.u. LDA exchangecorrelation after Ref. 25] is employed.

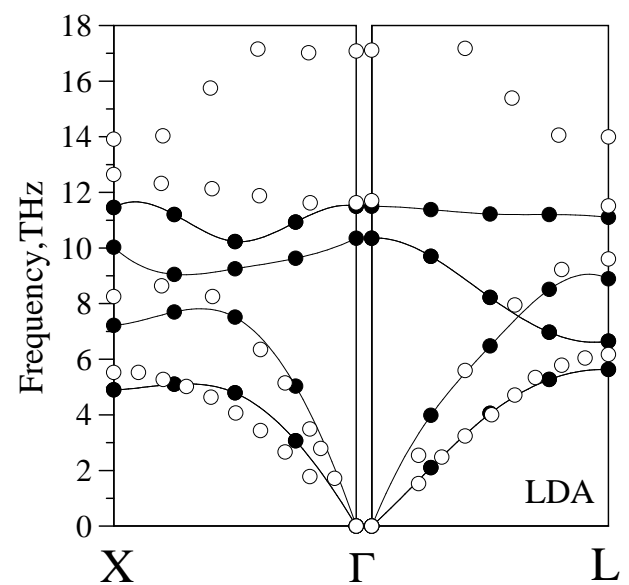

FigIIQ Savfasopar|komliaretween calculated using the LDA (filled circles) and experimental (open circles) phonon dispersion curves for $\mathrm{NiO}$.

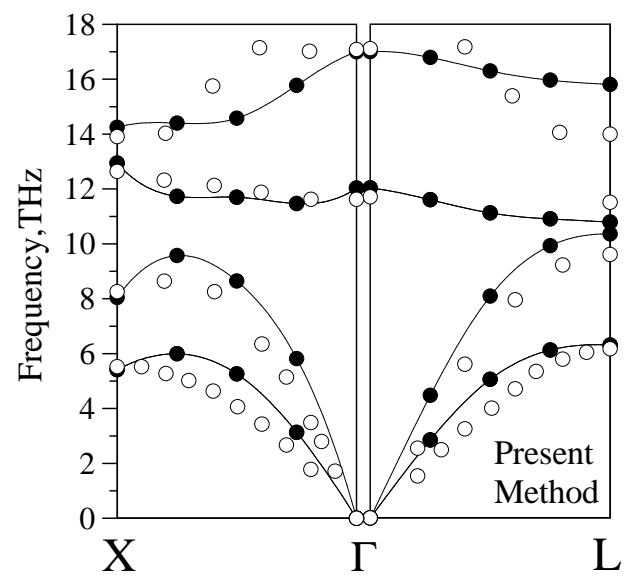

FIG. 2. Comparison between calculated using the present method (filled circles) and experimental (open circles) phonon dispersion curves for $\mathrm{NiO}$.

We first discuss the results of the LDA calculations for the lattice dynamics of NiO. Fig. 1 shows calculated phonon dispersion relations along major symmetry directions. Despite the unit cell corresponding to antiferromagnetic lattice contains two original unit cells, spin integrated properties are unaffected by this symmetry lowering and therefore can be represented in the original Brillouin zone. We see an apparent underestimation of all theoretical optical modes by as much as 50 or more per cent as compared to the experiment [26]. Similar finding for $\mathrm{MgO}$ has also been reported [7]. The $\mathrm{LO}-\mathrm{TO}$ splitting is largely underestimated which is predicted to be only $1.5 \mathrm{THz}$ against $5 \mathrm{THz}$ seen from the measurement. Surprisingly, however, that both longitudinal and transverse acoustic branches are much better predicted by the LDA. One can conclude that the calculated sound velocities along $\Gamma X$ direction are only slightly overestimated and the agreement is even better along the $\Gamma L$ line.

We now discuss how inclusion of correlations modifies the phonon spectrum. Fig. 2 shows the calculated dispersion curves using static self-energy approximation of the formalism developed in this work. We use the values of Hubbard $U=8 \mathrm{eV}$ and exchange $J=1 \mathrm{eV}$ in this calculation. We see a remarkable hardening of the optical modes which occurs due to proper treatment of the correlational effects. Notably both the values of the optical frequencies as well as the calculated LO-TO splitting are now in a good agreement with the experiment. A pronounced softening of the longitudinal optical mode along both $\Gamma X$ and $\Gamma L$ lines is seen at the measured data which is in part captured by our theoretical calculation: the agreement is somewhat better along the $\Gamma X$ direction while the detailed q-dependence of these branches shows some residual discrepancies.

Our acoustic modes are consistently overestimated by a few per cent as compared to the experiment. They are also harden as the on-site interaction $U$ increases although in much smaller degree than the optical modes. Overall, the agreement between the theory and the experiment is much better when the correlations are taken into account which indicates their importance in the lattice dynamics of strongly correlated materials.

To better understand our findings we have calculated the values of $\epsilon_{\infty}$ which represent an electronic contribution to the static dielectric constant as given by the inverse element $1 / \epsilon^{-1}(0,0)$ of the full inverse dielectric permittivity matrix in reciprocal space. Table 1 shows the results of these calculations using the LDA and our new method as well as the experiment 27]. It is seen that the LDA result overestimates the value of $\epsilon_{\infty}$ by a factor of 6 . This is mainly because the calculated by LDA dielectric gap value is only $0.4 \mathrm{eV}$ which is much smaller than the experimental energy gap of $\mathrm{NiO}$ equal to $4 \mathrm{eV}$ [28]. Namely LDA overestimates the electronic screening effects by a large amount causing both the artificial softening of optical phonons and the lowering of the LO-TO splitting. The latter is well known to be directly proportional to the Born effective charges but inversionally proportional to $\epsilon_{\infty}$.

On the other hand, our calculations with correlations produce a much better value of the static dielectric constant as seen from Table 1. We relate such an agreement with the fact that the energy gap value predicted by the theory here is $3.3 \mathrm{eV}$ which compares well with the measurement 28. This indicates that the screening effects 
in the calculation with correlations are treated in much more appropriate way which fixes both the position of the optical branches and their relative splitting.

As a final result, we have extracted the values of the Born effective charge $Z^{*}$ using $\mathbf{q} \rightarrow 0$ limit technique [29] and the relationship $\omega_{L O}^{2}-\omega_{T O}^{2} \propto\left|Z^{*}\right|^{2} / \epsilon_{\infty}$. Table 1 shows comparison between the theoretical and the experimental [26] data. The deviation from 2 which for binary oxides is the nominal rigid-ion value of $\left|Z^{*}\right|$ indicates high electronic polarizability. Both theoretical values are close to the experimental one, while the agreement for LDA is apparently accidental since the error in underestimating the splitting $\omega_{L O}^{2}-\omega_{T O}^{2}$ nearly completely cancels the error in overestimating $\epsilon_{\infty}$.

To summarize we have developed a new method to study the lattice dynamical properties of materials with strong electronic correlations. The method is based on the dynamical mean-field theory and it uses linear response technique to calculate phonons at arbitrary wave vector q. Using static approximation we have computed the phonon dispersions of $\mathrm{NiO}$ and find the results for the optical modes and static dielectric properties in much better agreement with the experiment than the results of the corresponding LDA based calculations. This emphasizes the importance of correlations in lattice dynamics of strongly correlated systems. The residual discrepancies can be attributed to the frequency dependence of the self-energy neglected in the present study and will be a subject for the future work. There are many other challenging problems for which the general framework outlined here can be useful. Discrepancies between theory and experiment were noticed 30 for the ferroelectric $\mathrm{CaCu}_{3} \mathrm{Ti}_{4} \mathrm{O}_{12}$. The electron-phonon interaction in cuprate superconductors is a subject of intensive investigation. Finally we believe that correlation effects play an important role in the phonon dynamics across the actinide series.

Acknowledgment: We are indebted to R. Resta and R. Cohen for valuable discussions, and the Division of Basic Energy Sciences of the US DOE for support under grant No. DE-FG02-99ER45761.

[1] For a review, see, e.g., Theory of the Inhomogeneous Electron Gas, edited by S. Lundqvist and S. H. March (Plenum, New York, 1983).

[2] For a review, see, e.g.,S. Baroni, S. de Gironcoli, A. Dal Corso, Rev. Mod. Phys. 73, 515-563 (2001);

[3] Baroni, S., P. Giannozzi, and A. Testa, Phys. Rev. Lett. 58, 1861 (1987);

[4] E. N. Zein, Fiz. Tverd. Tela (Leningrad) 26, 3024 (1984) [Sov. Phys. Solid State 26, 1825 (1984);

[5] For a review, see, e.g, Ab inition Calculations of Phonon
Spectra, edited by J. T. Devreese, V. E. Van Doren, and P. E. Van Camp (Plenum, New York, 1983);

[6] J. Bouchet, B. Siberchicot, F. Jollet, and A. Pasturel, J. Phys. Condens. Matter 12, 1723 (2000);

[7] S. Massidda, M. Posternak, A. Baldereschi, R. Resta, Phys. Rev. Lett. 82, 430 (1999);

[8] For a review, see, e.g., A. Georges, G. Kotliar, W. Krauth, and M. J. Rozenberg, Rev. Mod. Phys. 68, 13 (1996);

[9] V. I. Anisimov, A. I. Poteryaev, M. A. Korotin, A. O. Anokhin, G. Kotliar, J. Phys.: Condens. Matter 9, 7359 (1997);

[10] S. Lichtenstein, M. Katsenelson and G. Kotliar, Phys. Rev. Lett. 87, 067205 (2001).

[11] K. Held, A. K. McMahan and R. T. Scalettar, Phys. Rev. Lett. 87, 276404 (2001);

[12] S. Y. Savrasov, G. Kotliar, and E. Abrahams, Nature 410, 793-795 (2001);

[13] G. Kotliar and S. Savrasov, in New Theoretical approaches to strongly correlated systems, edited by A. M. Tsvelik (Kluwer Academic Publishers, The Netherlands, 2001); S. Y. Savrasov, G. Kotliar, cond-mat/0106308;

[14] For a review, see, V. I. Anisimov, F. Aryasetiawan, and A. I. Liechtenstein, J. Phys. Cond. Matter 9, 767 (1997);

[15] O. K. Andersen, Phys. Rev. B 12, 3060 (1975);

[16] S. Y. Savrasov, Phys. Rev. B 54, 16470 (1996);

[17] C.M. Varma, and W. Weber, Phys Rev. Lett. 39, 1094 (1977).

[18] X. Gonze, D. C. Allan, and M. P. Teter, Phys. Rev. Lett. 68, 3603 (1992);

[19] A. A. Quong and B. M. Klein, Phys. Rev. B 46, 10734 (1992);

[20] R.Yu, and H. Krakauer, Phys. Rev. B 49, 4467 (1994);

[21] R. Heid, and K.-P. Bohnen, Phys. Rev. B 60, R3709 (1999);

[22] S. Y. Savrasov, Phys. Rev. Lett. 69, 2819 (1992);

[23] P. Pulay, Mol. Phys. 17, 197 (1969);

[24] V. I. Anisimov, J. Zaanen, and O. K. Andersen, Phys. Rev. B 44, 943 (1991);

[25] S.H.Vosko, L.Wilk, and M.Nusair, Can. J. Phys.58, 1200 (1980);

[26] R.A. Roy, C. W. Tompson, and E. Gürmen, Sol. State. Comm. 18, 845 (1976);

[27] P. J. Gielisse, J. N. Plendel, L. C. Mansur, R. Marshall, S. S. Mitra, R. Mykolajewycz, A. Smakula, J. Appl. Phys. 36, 2446 (1965);

[28] G. A. Sawatzky, and J. W. Allen, Phys. Rev. Lett. 53 2339 (1984);

[29] C.-Z. Wang, R. Yu, and H. Krakauer, Phys. Rev. B 54, 11161 (1996);

[30] L. He, J.B. Neaton, M. H. Cohen, D. Vanderbilt, condmat/0110166.

TABLE I. Comparison between calculated static dielectric constant $\epsilon_{\infty}$ and Born effective charge $\left|Z^{*}\right|$ for NiO using LDA and present method as well as the experimental data [26,27]

\begin{tabular}{lccc}
\hline \hline & LDA & Present Method & Exp \\
\hline$\epsilon_{\infty}$ & 35.7 & 7.2 & $5.7,6.1$ \\
$\left|Z^{*}\right|$ & 2.17 & 2.33 & 2.22 \\
\hline \hline
\end{tabular}

